\title{
Weak pion-nucleon coupling strength: New constraint from parity mixing in ${ }^{18} \mathrm{~F}$
}

\author{
S. A. Page, ${ }^{*}$ H. C. Evans, G. T. Ewan, S. -P. Kwan, J. R. Leslie, J. D. MacArthur, \\ W. McLatchie, P. Skensved, S. -S. Wang, ${ }^{\dagger}$ and H. -B. Mak \\ Queen's University, Kingston, Ontario, Canada K7L $3 N 6$ \\ A. B. McDonald \\ Princeton University, Princeton, New Jersey 08544 \\ C. A. Barnes \\ California Institute of Technology, Pasadena, California 91125 \\ T. K. Alexander and E. T. H. Clifford ${ }^{\ddagger}$ \\ Atomic Energy of Canada Limited Research Company, Chalk River Nuclear Laboratories, Chalk River, Ontario, Canada K0J 1JO
}

(Received 28 October 1986)

\begin{abstract}
The circular polarization of the $1.081 \mathrm{MeV} \gamma$ rays from ${ }^{18} \mathrm{~F}$ has been measured to be $(1.7 \pm 5.8) \times 10^{-4} \cdot{ }^{18} \mathrm{~F}$ was produced by a $4.05 \mathrm{MeV}{ }^{3} \mathrm{He}^{+}$beam impinging on a recirculating water target with a thin entrance window. The circular polarization was measured with four magnetic transmission polarimeters, each backed by a $150 \mathrm{~cm}^{3} n$-type Ge detector. A fast data acquisition system limited overall peak losses to $30 \%$ at a count rate of $60 \mathrm{kHz}$ per detector. Supplementary experiments were carried out to investigate the sensitivity of the circular polarization measurement to systematic variations of beam position and intensity. These effects were found to be negligible relative to the statistical uncertainty. The experimental result is interpreted in terms of an isovector parity-nonconserving matrix element between the $\left|0^{+}, T=1,1.042 \mathrm{MeV}\right\rangle$ and $\left|0^{-}, T=0,1.081 \mathrm{MeV}\right\rangle$ states in ${ }^{18} \mathrm{~F}$ that is dominated by the weak pion exchange contribution. The present result for the weak pion-nucleon coupling strength is $\left|f_{\pi}^{1}\right|=\left(0.4_{-0.4}^{+1.4}\right) \times 10^{-7}$, which is significantly smaller than recent theoretical predictions based on the Weinberg-Salam model.
\end{abstract}

\section{INTRODUCTION}

Although evidence of parity nonconservation (PNC) from observation of electromagnetic decays of nuclear states has been reported for nearly two decades, ${ }^{1}$ the weak nucleon-nucleon (N-N) interaction is not quantitatively understood. The calculation of matrix elements of the weak $\mathrm{N}-\mathrm{N}$ potential needed to relate PNC observables to parameters of the weak hadronic interaction introduces large uncertainties when, as in most cases, inadequate nuclear wave functions are used. These theoretical problems are most severe for heavy nuclei, in which some of the largest PNC effects have been observed. There are, however, examples involving light nuclei in which a paritymixed doublet of states is found at low excitation energy, the PNC signal is enhanced by nuclear structure, and the nuclear matrix elements can be more reliably calculated and verified by comparison with analog $\beta$-decay matrix elements. A particularly favorable case is that of ${ }^{18} \mathrm{~F}$, in which the PNC matrix element between the $0^{+}, 1.042$ $\mathrm{MeV}$ and $0^{-}, 1.081 \mathrm{MeV}$ states can be determined in a manner that is virtually independent of nuclear structure assumptions and leads to an unambiguous evaluation of the weak pion-nucleon coupling strength.

At low energies $(E \leq 300 \mathrm{MeV})$, the weak N-N interaction is described in terms of an effective meson exchange model in which a meson is coupled to a nucleon via the weak interaction at one vertex and the strong interaction at the other. The weak N-N interaction does not conserve isospin or parity but does conserve CP; as a result neutral pseudoscalar meson (e.g., $\pi^{0}, \eta$ ) exchanges are excluded from the model. ${ }^{2}$ The most comprehensive theoretical treatment to date is that of Desplanques, Donoghue, and Holstein $^{3}(\mathrm{DDH})$, who parametrized the weak N-N potential $V^{\mathrm{PNC}}$ in terms of $\pi, \rho$, and $\omega$ exchanges and calculated the coupling constants of six weak meson-nucleonnucleon vertices using the standard electroweak theory ${ }^{4}$ and a quark model of the mesons and nucleons. A number of the parameters of the model were determined by comparison with strangeness changing hadronic weak decay lifetimes. However, contributions from $Z^{0}$ exchanges between quarks are suppressed in these cases and can only be determined from the $\Delta S=0$ weak meson-nucleonnucleon vertices.

Because of uncertainties inherent in the quark model calculations, DDH reported their calculations in terms of broad "reasonable ranges" and "best estimates" of the weak N-N interaction coupling constants $f_{\pi}^{1}, h_{\omega}^{0}, h_{\omega}^{1}, h_{\rho}^{0}$, $h_{\rho}^{1}$, and $h_{\rho}^{2}$ (the superscripts label the isospin character of the effective interaction). The determination of these weak meson-nucleon coupling constants from experiment is an essential test of the weak meson exchange model. To this end, it is clearly advantageous to determine the individual contributions to $V^{\mathrm{PNC}}$ separately. The weak pionexchange potential contributes only to isovector PNC processes, and as the pion is relatively light, its contribution 
is expected to dominate the weak isovector $\mathrm{N}-\mathrm{N}$ interaction at low energy.

A partial energy level scheme of ${ }^{18} \mathrm{~F}$ is shown in Fig. 1. The isovector component of $V^{\mathrm{PNC}}$ is expected to cause opposite parity admixtures in the wave functions of the $\left(0^{+}, 1\right)$ and $\left(0^{-}, 0\right)$ states at 1.042 and $1.081 \mathrm{MeV}$, respectively. The nearest spin- 0 state is at $4.75 \mathrm{MeV}$, which justifies the approximation of two-level mixing. The wave functions of the 1.042 and $1.081 \mathrm{MeV}$ states may be expressed as

$$
|1.042\rangle=\left|0^{+}, 1\right\rangle-\epsilon\left|0^{-}, 0\right\rangle
$$

and

$$
|1.081\rangle=\left|0^{-}, 0\right\rangle+\epsilon\left|0^{+}, 1\right\rangle,
$$

with the mixing parameter $\epsilon<1$ given by

$$
\epsilon=\frac{\left\langle 0^{+}, 1\left|V^{\mathrm{PNC}}\right| 0^{-}, 0\right\rangle}{\delta E}
$$

and the separation $\delta E$ was recently determined ${ }^{5}$ to be $39.20 \pm 0.11 \mathrm{keV}$. The PNC matrix element which is sensitive to $\pi, \rho$, and $\omega$ exchanges can in this case be written as

$$
\begin{aligned}
\left\langle 0^{+}, 1\left|V^{\mathrm{PNC}}\right| 0^{-}, 0\right\rangle & \\
& =\left\langle 0^{+}, 1\left|\left(f_{\pi}^{1} V_{\pi}^{1}+h_{\rho}^{1} V_{\rho}^{1}+h_{\omega}^{1} V_{\omega}^{1}\right)\right| 0^{-}, 0\right\rangle,
\end{aligned}
$$

and is expected to be dominated by the long range pion exchange contribution.

Here, the PNC observable is the circular polarization of the $\gamma$ rays emitted in the decays of the parity-mixed states, ${ }^{18} \mathrm{~F}(1.042 \mathrm{MeV} \rightarrow$ g.s. $)$ and ${ }^{18} \mathrm{~F}(1.081 \mathrm{MeV} \rightarrow$ g.s. $)$, which are predominantly magnetic dipole $(M 1)$ and electric dipole $(E 1)$ transitions, respectively. To first order in $\epsilon$, the circular polarizations of the 1.042 and $1.081 \mathrm{MeV} \gamma$

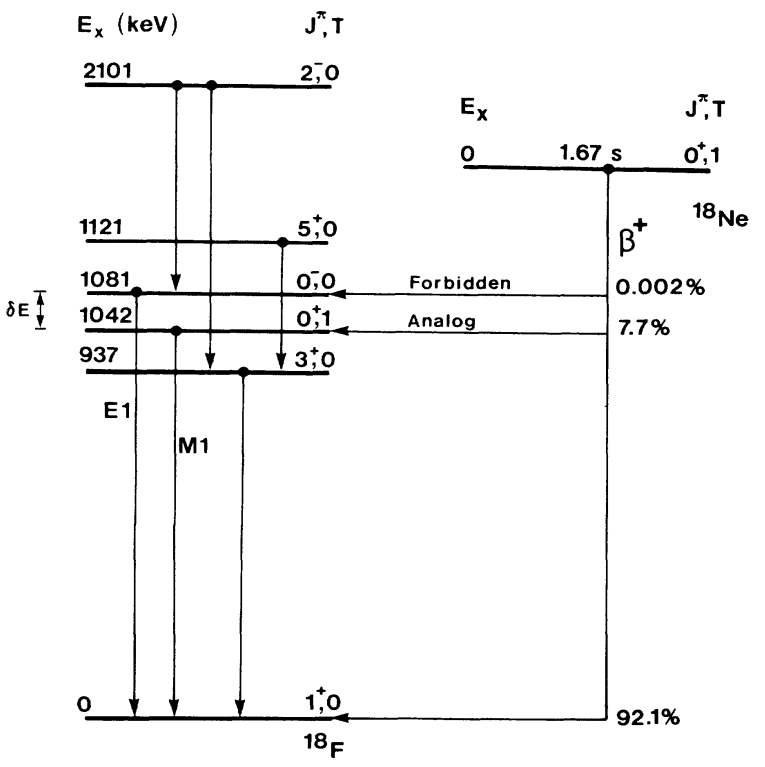

FIG. 1. Partial energy level diagram of ${ }^{18} \mathrm{~F}$, showing levels relevant to the present experiment. The energy spacing is not to scale. rays are given by

$P_{\gamma}(1.042)$

$$
=-\frac{2}{\delta E} \operatorname{Re}\left(\frac{\left\langle 0^{+}, 1\left|V^{\mathrm{PNC}}\right| 0^{-}, 0\right\rangle\left\langle 1^{+}|| E 1 \| 0^{-}\right\rangle}{\left\langle 1^{+}\|M 1\| 0^{+}\right\rangle}\right)
$$

and

$$
\begin{aligned}
& P_{\gamma}(1.081) \\
& \quad=\frac{2}{\delta E} \operatorname{Re}\left(\frac{\left\langle 0^{+}, 1\left|V^{\mathrm{PNC}}\right| 0^{-}, 0\right\rangle\left\langle 1^{+}\|M 1\| 0^{+}\right\rangle}{\left\langle 1^{+}|| E 1 \| 0^{-}\right\rangle}\right),
\end{aligned}
$$

where $P_{\gamma}$ is taken to be positive for a right-handed photon. The ratio of reduced matrix elements of the $M 1$ and $E 1$ operators is determined from the measured lifetimes ${ }^{6}$ of the 1.042 and $1.081 \mathrm{MeV}$ states. This factor,

$$
\begin{aligned}
\left|R_{m}\right| & =\left|\frac{\left\langle 1^{+}\|M 1\| 0^{+}\right\rangle}{\left\langle 1^{+}\|E 1\| 0^{-}\right\rangle}\right| \\
& =\left[\frac{\tau(1.081)}{\tau(1.042)}\right]^{1 / 2}\left[\frac{1.081}{1.042}\right]^{3 / 2}=110 \pm 10,
\end{aligned}
$$

enhances the circular polarization of the $1.081 \mathrm{MeV} \gamma$ rays by $\simeq 10^{4}$ with respect to the $1.042 \mathrm{MeV} \gamma$ rays. The basis of this favorable enhancement is the strong suppression of the $\Delta I=0 E 1$ matrix element, which effectively precludes a reliable prediction of the sign of $R_{m}$.

In order to deduce a value for $f_{\pi}^{1}$ from a measurement of $P_{\gamma}(1.081)$ in ${ }^{18} \mathrm{~F}$, two-body matrix elements of the weak isovector $\pi, \rho$, and $\omega$ exchange potentials must be calculated. ${ }^{18} \mathrm{~F}$ is a fortunate case in which the PNC matrix element $\left\langle 0^{+}, 1\left|V_{\pi}^{1}\right| 0^{-}, 0\right\rangle$ can be "calibrated" by a measurement of the forbidden $\beta^{+}$-decay rate ${ }^{18} \mathrm{Ne}$ (g.s.) $\rightarrow{ }^{18} \mathrm{~F}(1.081 \mathrm{MeV})$. The two-body pion-exchange contribution to this $\beta$-decay strength is due to an operator which is related to the PNC pion exchange potential by an isospin rotation. ${ }^{7}$

A detailed study was recently reported by Adelberger et al.,$^{5}$ in which the one- and two-body contributions to the $\beta$-ray transition were calculated with four sets of shell model wave functions, differing in the size of the model space and the form of the residual interactions. These calculations were compared with the measured $\beta$-decay rate $\left(\omega_{\beta}\right)$ of ${ }^{18} \mathrm{Ne}$ to the $1.081 \mathrm{MeV}$ state in ${ }^{18} \mathrm{~F}$. Although absolute predictions of $\omega_{\beta}$ varied by an order of magnitude for the different wave functions of this study, in all cases the predicted ratios of two- and one-body contributions were found to be within the range $0.73 \pm 0.10$. This result, together with the measured $\beta^{+}$-decay rate, ${ }^{5,8}$ leads to a virtually model-independent prediction of the weak pion exchange matrix element in ${ }^{18} \mathrm{~F}$. In addition, Adelberger et al. ${ }^{5}$ found consistent relative contributions of isovector $\pi, \rho$, and $\omega$ exchanges despite wide variations in their absolute contributions. With the DDH best values of $f_{\pi}^{1}, h_{\rho}^{1}$, and $h_{\omega}^{1}$, the circular polarization of the 1.081 $\mathrm{MeV} \gamma$ rays is predicted to be $\left|P_{\gamma}(1.081)\right|=2.1 \times 10^{-3}$. The $\rho$ and $\omega$ exchange contributions were found to be small ( $\leqslant 5 \%$ using the DDH best values) and to add constructively in the matrix element responsible for parity mixing in ${ }^{18} \mathrm{~F}$. Hence the measured value of $P_{\gamma}(1.081)$ 
can be used to set an upper bound for the weak pionnucleon coupling $f_{\pi}^{1}$. By scaling the formula of Adelberger et al. ${ }^{5}$ with the weighted average of measured decay rates, $\omega_{\beta}=(8.6 \pm 1.2) \times 10^{-6} \mathrm{~s}^{-1}$, the following expression is obtained,

$$
\left|f_{\pi}^{1}\right|=(2.31 \pm 0.43) \times 10^{-4}\left|P_{\gamma}(1.081)\right|,
$$

and $f_{\pi}^{1}$ is taken to be positive on theoretical grounds. ${ }^{3}$

The weighted average of the previous measurements ${ }^{9-11}$ of the circular polarization $P_{\gamma}(1081)$ $=(-0.8 \pm 1.2) \times 10^{-3}$ places an upper bound on the value of $f_{\pi}^{1}$ close to the theoretical best value of $4.6 \times 10^{-7}$. The present paper describes an experiment in which the uncertainty in $P_{\gamma}$ has been reduced by a factor of 2 . It was anticipated that the present work would permit the extraction of a more precise value for $f_{\pi}^{1}$. Bini et al. ${ }^{12}$ have also recently reported a comparable value of $P_{\gamma}$. Improvements in the present experiment include a four Ge-detector symmetric $\gamma$-ray circular polarimeter designed to minimize the effect of stray magnetic fields on the beam, an amplifier system capable of counting at $60 \mathrm{kHz}$ with good energy resolution, an analog-to-digital converter (ADC) multiplexer which reduced ADC dead times to a negligible level, and a Ti foil window water target and extended counting times. Internal checks and auxiliary experiments have been performed to estimate the size of possible systematic effects.

\section{EXPERIMENTAL PROCEDURE}

\section{A. Recirculating water target}

Excited ${ }^{18} \mathrm{~F}$ nuclei were produced in the reaction ${ }^{16} \mathrm{O}\left({ }^{3} \mathrm{He}, \mathrm{p}\right){ }^{18} \mathrm{~F}$ at the Queen's University Van de Graaff accelerator laboratory. The $4.05 \mathrm{MeV}{ }^{3} \mathrm{He}^{+}$beam passed through a thin $\mathrm{Ti}$ foil entrance window $\left(0.9-1.1 \mathrm{mg} / \mathrm{cm}^{2}\right.$, $\Delta E=0.4-0.5 \mathrm{MeV}$ (see Fig. 2) and impinged upon a recirculating water target. The beam energy was chosen to maximize the ${ }^{18} \mathrm{~F}$ yield while remaining below the ${ }^{16} \mathrm{O}\left({ }^{3} \mathrm{He}, \mathrm{n}\right)$ threshold to reduce the neutron induced $\gamma$-ray background and the possibility of neutron damage to the Ge detectors. At this energy, the $\mathrm{Ti}$ target windows normally withstood beam currents of $10-15 \mu \mathrm{A}$ for $48 \mathrm{~h}$, a significant improvement over the performance of the $\mathrm{Ni}$ entrance foils used in some of the earlier measurements. ${ }^{9,11}$ There was no evidence of $\gamma$ rays resulting from ${ }^{3} \mathrm{He}$ induced reactions on $\mathrm{Ti}$. The foils were attached to

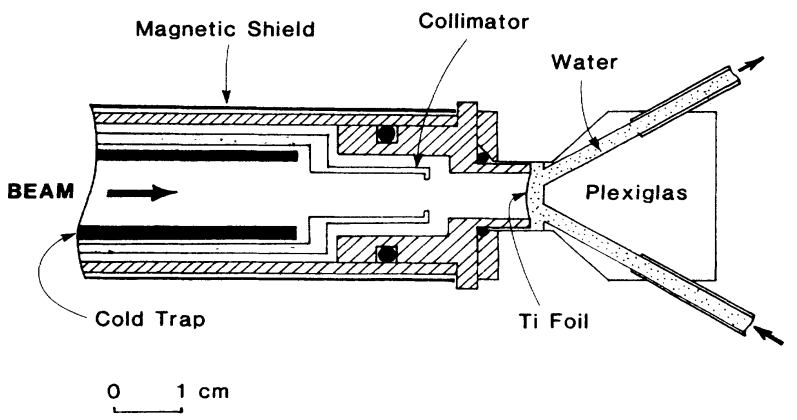

FIG. 2. Cross-sectional view of the water target system used in the present experiment. axially symmetric stainless steel holders with Armstrong A-12 epoxy; a circular area of foil $0.63 \mathrm{~cm}$ in diameter was exposed to the beam. To minimize the production of neutrons from the reaction ${ }^{12} \mathrm{C}\left({ }^{3} \mathrm{He}, \mathrm{n}\right){ }^{14} \mathrm{O}$, which occurred because of the gradual buildup of ${ }^{12} \mathrm{C}$ on the window foils, the target foils were stored in a clean pumping station and outgassed at $\simeq 100^{\circ} \mathrm{C}$ for $4-6 \mathrm{~h}$ prior to installation in the beam line.

The yield of ${ }^{14} \mathrm{~N} \gamma$ rays at $2.31 \mathrm{MeV}$, produced in the reaction ${ }^{12} \mathrm{C}\left({ }^{3} \mathrm{He}, \mathrm{p}\right){ }^{14} \mathrm{~N}$, was monitored as an indication of the amount of ${ }^{12} \mathrm{C}$ exposed to the beam. During the 2-3 d beam exposure for a typical foil, the $2.31 \mathrm{MeV} \gamma$ ray yield increased to $10 \%$ of the $1.081 \mathrm{MeV}$ yield; the total count rate would have increased by as much as $30 \%$ for a constant beam current, and the $1.081 \mathrm{MeV}$ peak-tobackground ratio decreased by approximately $20 \%$. The increase in count rate was thought to be due to a reduction in foil thickness by sputtering and hence an increase in the effective beam energy on target. To maintain a fixed event rate of $60 \mathrm{kHz}$ in each detector, the beam current was decreased gradually.

\section{B. Polarimeters}

The $\gamma$-ray asymmetry $A_{\gamma}$ is defined by

$$
A_{\gamma}=\frac{N^{+}-N^{-}}{N^{+}+N^{-}} \text {, }
$$

where $N^{+}\left(N^{-}\right)$denotes the number of $\gamma$ rays transmitted through a magnetic polarimeter when the electron polarization is parallel (antiparallel) to the photon propagation direction. The asymmetry $A_{\gamma}$ is related to the $\gamma$-ray circular polarization $P_{\gamma}$ by

$$
A_{\gamma}=P_{\gamma} \tanh \left(-\sigma_{c} n_{\mathrm{e}} \nu_{\mathrm{e}} L^{\prime}\right) \equiv \eta P_{\gamma},
$$

where the circular polarization sensitivity $\eta$ is determined by the circular polarization dependent contribution to the Compton scattering cross section ${ }^{13} \sigma_{c}$, the electron density $n_{\mathrm{e}}$, the electron polarization $v_{\mathrm{e}}$, and the effective polarimeter length $L^{\prime}$, which is less than the physical length due to the nonaxial orientation of the magnetic fields at the ends of the polarimeter.

The iron-cobalt alloy Permendur $(49 \% \mathrm{Fe}, 49 \% \mathrm{Co}$, $\left.2 \% \mathrm{~V} ; n_{\mathrm{e}}=2.28 \times 10^{24} \mathrm{~cm}^{-3} ; B_{\text {sat }}=2.35 \mathrm{~T}\right)$ was selected for the polarimeter cores and Armco iron was used for the flux returns. The polarimeter length was chosen to be 7.2 $\mathrm{cm}$, based on count rate tests with an iron absorber and a conservative estimate of $10 \mu \mathrm{A}$ for the maximum beam current that the Ti foils could withstand. The shape of the polarimeters was designed to minimize stray magnetic fields along the beam trajectory while maximizing the effective polarimeter length $L^{\prime}$. This was done using the magnetic field program TRIM at Chalk River Nuclear Laboratories. ${ }^{14}$ The design is shown in Fig. 3. Based on properties of a similar polarimeter reported in the literature, ${ }^{15}$ the effective length $L^{\prime}$ is calculated to be $6.4 \pm 0.4$ cm.

Four identical polarimeters of this design were constructed. The magnetizing coils consisted of 607 turns of no. 19 wire; the polarimeter cores were cooled by water flowing in a $2 \mathrm{~mm} \mathrm{Cu}$ tube encircling the back ends of the 

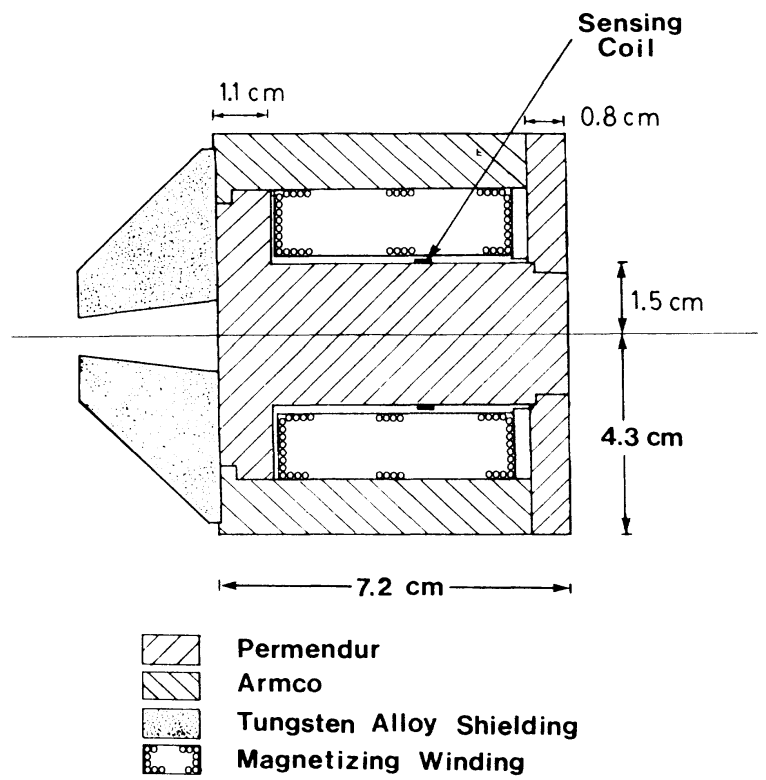

FIG. 3. Cross section of a polarimeter.

polarimeters. A four turn sensing coil was wound around the core of each polarimeter and connected to an integrating amplifier circuit. During data acquisition, the polarity of the 3 A magnetizing current was reversed by an optically coupled switching bridge. The risetime of the integrated sense coil pulses indicated that the field switching time at $3 \mathrm{~A}$ was less than $50 \mathrm{~ms}$. The magnetic fields in the cores were found to be $2.31 \pm 0.06 \mathrm{~T}$, in agreement with the manufacturer's specifications for Permendur.

The four polarimeters were placed symmetrically about the target at $90^{\circ}$ to the beam direction, as shown in Fig. 4 .

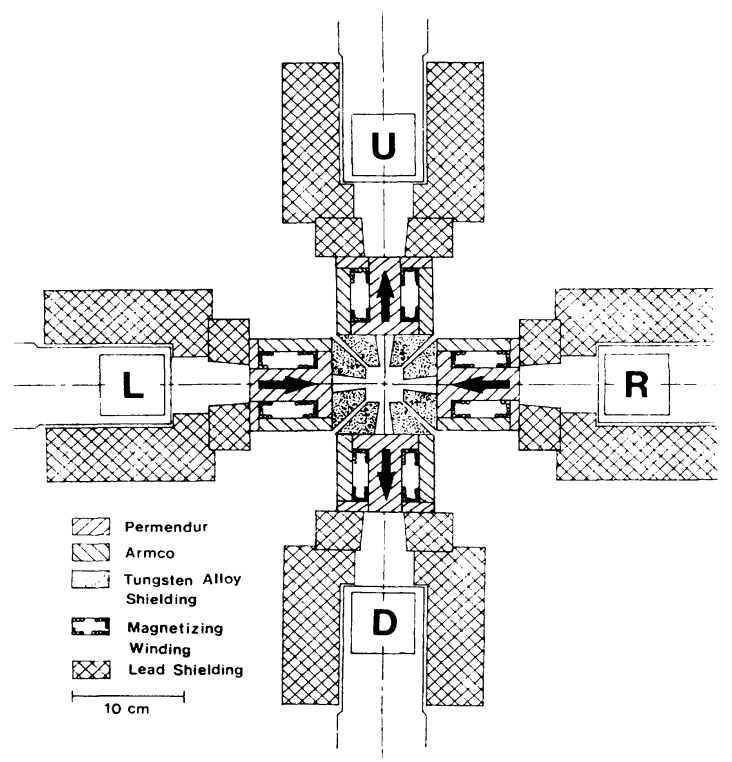

FIG. 4. View of the polarimeter assembly. The up, down, left, and right detectors are labeled $U, D, L$, and $R$, respectively. The arrows on the polarimeter cores indicate the magnetization direction in polarimeter state $(0)$.
With the magnetic fields in the polarimeters as shown, residual fields are expected to cancel along the beam axis if the polarimeters are identical. Residual magnetic fields were mapped along the central axis $(z)$ of the beamline to $50 \mathrm{~cm}$ upstream from the target using a Hall effect probe, before and after two concentric isolated layers of $\mathrm{Co}-$ Netic high permeability magnetic shielding were installed around the last $40 \mathrm{~cm}$ of beamline. A $z^{-3}$ (magnetic dipole) dependence of the residual fields was assumed for the range $50<z<350 \mathrm{~cm}$, as the magnetic fields were barely detectable with the Hall probe $\left(<10^{-7} \mathrm{~T}\right)$ beyond $50 \mathrm{~cm}$ upstream from the target. The systematic deflection of a $4.05 \mathrm{MeV}{ }^{3} \mathrm{He}^{+}$beam was calculated numerically and the results are summarized in Table I. With the calculated field dependent deflection of $0.4 \times 10^{-5} \mathrm{~cm}$ in the target-to-detector distance of $19.5 \mathrm{~cm}$ and a pessimistic estimate of $0.5 \mathrm{~cm}$ for the beam offset from the center of the polarimeter assembly, the spurious asymmetry caused by beam steering in the residual polarimeter fields is predicted to be $\delta A_{\gamma}<1 \times 10^{-8}$.

\section{Detectors}

Each magnetic polarimeter was backed by an Ortec intrinsic $n$-type closed-end coaxial Ge detector. Detector efficiencies at $1333 \mathrm{keV}$ were $30 \%$ relative to a 7.6 $\mathrm{cm} \times 7.6 \mathrm{~cm} \mathrm{NaI}$ detector and the spread in the efficiencies was less than $5 \%$ of this value. The Ge crystal locations were mapped with a collimated ${ }^{60} \mathrm{Co} \gamma$-ray source, and the detectors were positioned behind the polarimeters with the fronts of the crystals equidistant from the center of the target.

The tungsten alloy entrance collimators (shown in Fig. 3) fixed to the polarimeters improved the peak-tobackground area ratio at $1.081 \mathrm{MeV}$ by $\simeq 40 \%$. In a preliminary experiment, the position of the target along the beam axis was determined by a scan of the $0.937 \mathrm{MeV} \gamma-$ ray yield as the target was moved in that direction; subsequently, guides ensured the reproducibility of the target location to within $0.5 \mathrm{~mm}$.

\section{The data acquisition system}

Data acquisition for the experiment was controlled by a microprocessor-based timing sequencer unit (TS) interfaced to a PDP-15 computer. The TS was programmed to repeat a fixed set of instructions for a preset time period during which data were accumulated and stored in the computer memory. A typical program cycle consisted of $10 \mathrm{~s}$ data accumulation in one polarimeter state, then $100 \mathrm{~ms}$ in which data accumulation was halted and the direction of the magnetizing current in the polarimeter coils was reversed, followed by an equal counting period

TABLE I. ${ }^{3} \mathrm{He}^{+}$beam deflection due to residual polarimeter fields.

\begin{tabular}{lcc}
\hline \hline \multicolumn{1}{c}{ Direction } & $\begin{array}{c}\text { Without shielding } \\
(\mathrm{m})\end{array}$ & $\begin{array}{c}\text { With shielding } \\
(\mathrm{m})\end{array}$ \\
\hline Vertical $(\Delta x)$ & $-3.7 \times 10^{-7}$ & $-0.2 \times 10^{-7}$ \\
Horitontal $(\Delta y)$ & $+3.0 \times 10^{-7}$ & $+0.3 \times 10^{-7}$ \\
\hline \hline
\end{tabular}


in the other polarimeter state. A typical 15 min run consisted of a sequence of 40 cycles of the above program, at the end of which data accumulation was halted and the $\gamma$-ray spectra and timer and scaler information were written onto magnetic tape.

To perform the circular polarization measurement to the intended accuracy, a data acquisition system ${ }^{16}$ capable of handling event rates in the range $50-70 \mathrm{kHz}$ per detector with minimal losses due to dead time and pileup was built. To reduce pileup losses, Ortec 673 gated integrating amplifiers (GI's) with $0.25 \mu \mathrm{s}$ shaping time were used. With this time constant, the throughput peak count loss was $\sim 30 \%$ at a detector count rate of $60 \mathrm{kHz}$, and the resolution at $1.333 \mathrm{MeV}$ was $2.8 \mathrm{keV}$ full width at half maximum (FWHM). This was a small degradation of the $2.2 \mathrm{keV}$ FWHM resolution obtained at a detector count rate of $2 \mathrm{kHz}$ and $2 \mu$ s time constant.

At an incident beam energy of $4.05 \mathrm{MeV}$, the ${ }^{16} \mathrm{O}\left({ }^{3} \mathrm{He}, \mathrm{p}\right)$ reaction populates states in ${ }^{18} \mathrm{~F}$ up to $5 \mathrm{MeV}$ in excitation. For the circular polarization measurement, $\gamma$ ray events in the energy range $0.8-1.4 \mathrm{MeV}$ were selected for analysis, corresponding to $\simeq 25 \%$ of the total detector count rate. These events were selected from the unipolar pulse outputs of the GI's by single-channel analyzers (SCA's). The SCA logic signals were used to trigger the linear gates through which GI signals in the desired energy range were delayed and sent to three $200 \mathrm{MHz}$ ADC's via a data multiplexing system (MUX) which assigned events from all four detectors to one of the three ADC's and recorded events lost due to ADC deadtimes. For each event, the MUX sent logic signals identifying the detector, ADC, and polarimeter state to the PDP-15 computer to determine in which one of 24 1024-channel spectra the event was to be stored. If all three ADC's were busy when an SCA pulse arrived, the event was lost and the missed count scaler (MCS) was incremented.

To test the performance of the data multiplexing system, spectra were accumulated from ${ }^{60} \mathrm{Co}$ sources at the same count rates as in the ${ }^{18} \mathrm{~F}$ experiment. The MUX was gated off in one polarimeter state, and the number of misrouted events, which appeared at random locations in the spectra for this polarimeter state, was observed. The maximum possible effect of misrouting deduced from these measurements was $\delta A_{\gamma}=1 \times 10^{-7}$, two orders of magnitude smaller than the statistical accuracy to which the asymmetry was measured.

\section{E. Measurement of $P_{\gamma}(1.081)$}

In the absence of systematic effects, the eightfold ratio $\xi$ is related to the $\gamma$-ray circular polarization $P_{\gamma}$ by

$$
\begin{aligned}
\xi & =\frac{U(0) D(0) L(1) R(1)}{U(1) D(1) L(0) R(0)} \\
& =\left|\frac{1-A_{\gamma}}{1+A_{\gamma}}\right|^{4} \simeq 1-8 A_{\gamma}=1-8 \eta P_{\gamma},
\end{aligned}
$$

where $U(0)$ denotes the number of $\gamma$ rays in detector $U$ in polarimeter state $(0)$, for the field configuration shown in Fig. 4. To first order, this ratio, $\xi$, is insensitive to differences in detector efficiencies, the dead times of the sys- tem, and the total charge accumulated in the two polarimeter states.

Gamma-ray spectra from ${ }^{18} \mathrm{~F}$ were accumulated in a series of 15-min runs for $2560 \mathrm{~h}$. A typical spectrum from one detector is shown in Fig. 5. To minimize systematic bias in the experiment, the length of the counting intervals in the two field states was varied between 7-17 s in different runs. In addition, the relationship between logic levels and the direction of the current in the magnetizing coils was reversed for alternate data tapes (180 runs) to cancel possible systematic effects related to the TS unit.

An on-line analysis of the spectra was performed to monitor event ratios sensitive to beam steering and beam intensity fluctuations which could affect the quality of the data. The beam current of $10-15 \mu \mathrm{A}$ on target was adjusted to keep the detector count rates in the optimum range of $60-65 \mathrm{kHz}$. The sense coil signals were monitored with digital voltmeters to check the consistency of the magnetic fields in the polarimeter cores. The position of the beam on the target, as indicated by a bright source of light in the water, was monitored with a television system, and beam steering magnets were adjusted to keep the beam spot centered on the target.

\section{F. Measurement of the circular polarization sensitivity}

The circular polarization sensitivity $\eta$ was measured with two of the polarimeters in series, as shown in Fig. 6. The polarimeters remained in the same configuration as in the ${ }^{18} \mathrm{~F}$ experiment, and the $R$ detector was replaced by a $0.5 \mathrm{Ci}{ }^{60} \mathrm{Co}$ source surrounded by lead shielding. Unpolarized $\gamma$ rays from the source acquired circular polarization $\eta$ after passing through the $R$ polarimeter in which the magnetic field was held constant. This polarization was then analyzed by the $L$ polarimeter in which the magnetic field direction was reversed every $10 \mathrm{~s}$; the twofold ratio of counts in the $L$ detector, $R_{\eta}$, is related to the circular polarization sensitivity by

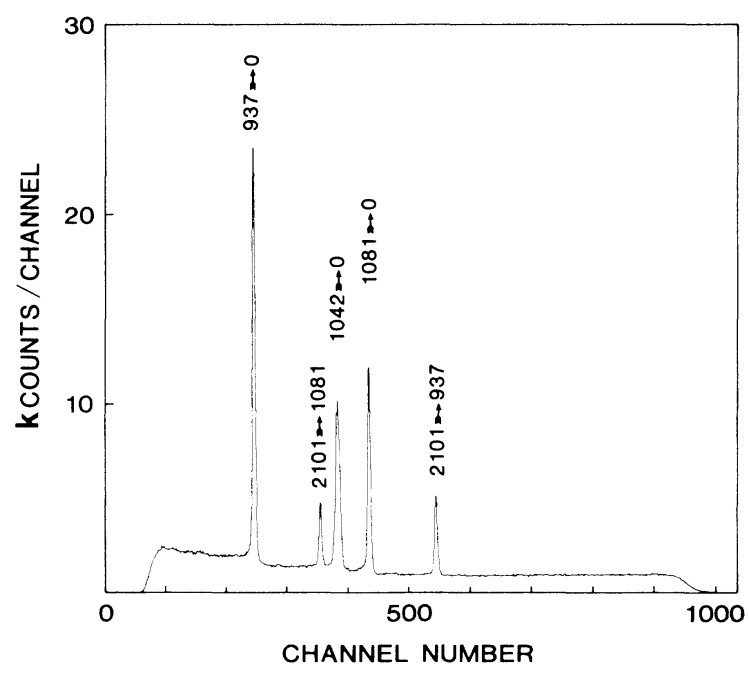

FIG. 5. A typical spectrum from one detector. 


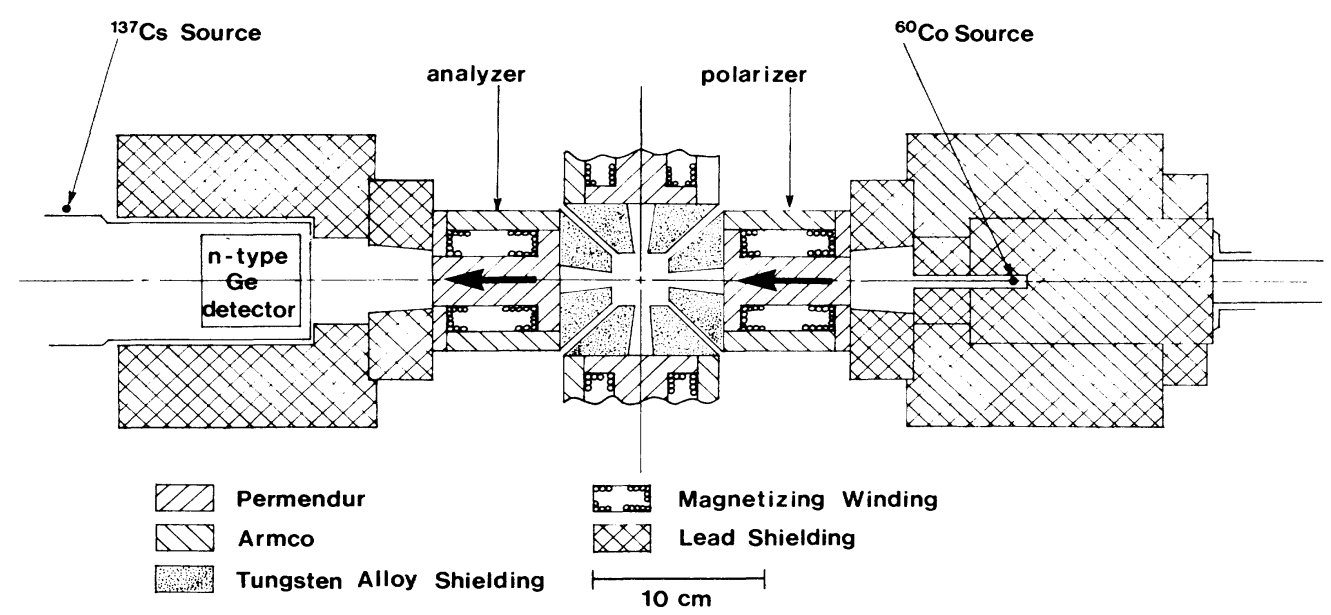

FIG. 6. Setup of the polarimeter calibration experiment. Arrows indicate the magnetic field directions in state (0).

$$
R_{\eta}=\frac{L(0)}{L(1)}=1+2 \eta^{2}
$$

for the configuration shown in Fig. 6. A ${ }^{137} \mathrm{Cs}$ source was placed near the $L$ detector to monitor the counting rate in the two polarimeter states. During the data accumulation period of $20 \mathrm{~d}$, the direction of the polarizing field was periodically reversed.

The ratio $R_{\eta}$ was calculated for the 1.173 and 1.332 $\mathrm{MeV} \gamma$ rays from ${ }^{60} \mathrm{Co}$ and normalized to the value obtained for the $0.662 \mathrm{MeV}$ line from the ${ }^{137} \mathrm{Cs}$ source. The measured values of $\eta(1.173)$ and $\eta(1.332)$ were scaled by the energy dependence ${ }^{13}$ of $\sigma_{c}$ to deduce $\eta(1.081)$. The circular polarization sensitivity $\eta(1.081)$ was found to be $0.0161 \pm 0.0008$, in agreement with the value of $0.017 \pm 0.002$, predicted from the effective polarimeter length $L^{\prime}$ discussed in Sec. II B.

\section{ANALYSIS}

\section{A. Determination of the asymmetries $A_{\gamma}$}

The asymmetries $A_{\gamma}$ for the five backgroundsubtracted peak areas of the spectra were determined by a method which minimized the uncertainties $\delta A_{\gamma}$. This was achieved by varying the integration limits in the calculation of $\gamma$-ray peak areas from which $\xi$ was determined. Qualitatively, the uncertainty is expected to be larger for integration limits which are extremely narrow or extremely wide, corresponding to the exclusion of peak counts from the integral and the inclusion of unnecessary background events, respectively.

To determine the optimum integrating width, spectra from all acceptable runs were added together, with gain and baseline shift corrections applied identically in both polarimeter states, to produce a master spectrum for each detector in each polarimeter state. Runs were rejected if the asymmetry in the charge deposited on the target $\left|A_{q}\right|$ was greater than 0.0025 , where

$$
A_{q}=\frac{Q(0)}{Q(1)}-1
$$

and $Q(0)$ is the charge deposited on the target in state $(0)$ accumulated by a scaler in the TS. This criterion reduced the data base by $1.7 \%$; the charge asymmetry $A_{q}$ for the accepted data was $-4 \times 10^{-5}$.

Asymmetries were calculated for the five $\gamma$-ray lines from the master spectra as a function of integration width

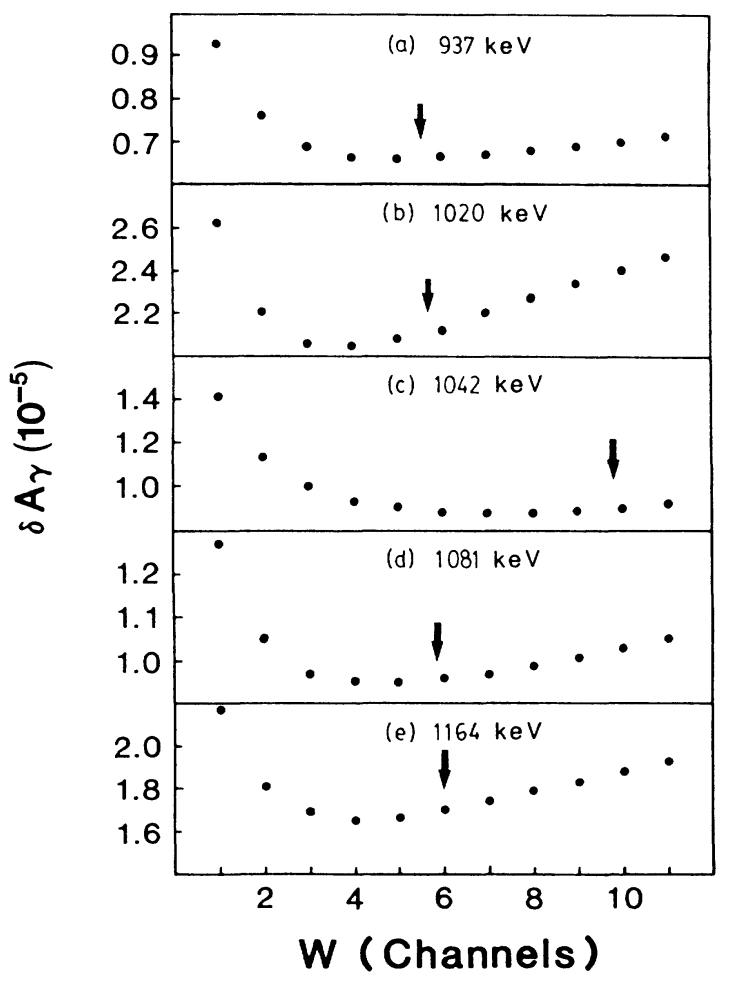

FIG. 7. Statistical uncertainty in the $\gamma$-ray asymmetry as a function of integration width $W$ for the five $\gamma$ rays from ${ }^{18} \mathrm{~F}$, deduced from the master spectrum. Limits of integration are from $P-W$ to $P+W$, where $P$ is the centroid of a $\gamma$-ray peak. The arrows indicate the integration limits used in the final data analysis. 


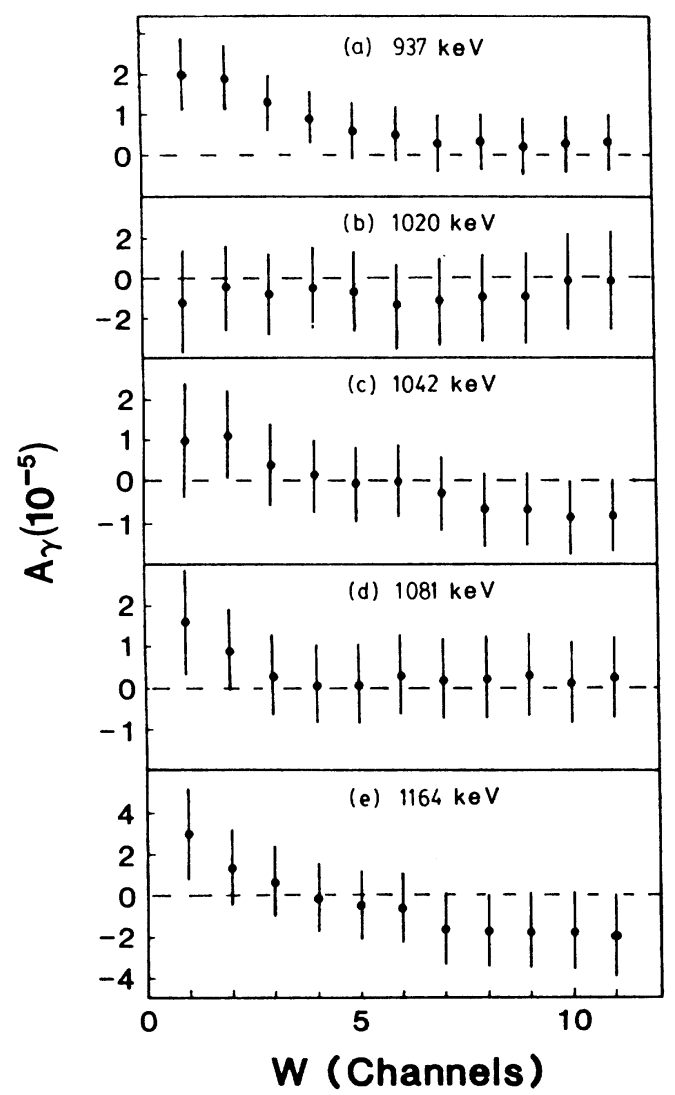

FIG. 8. Gamma-ray asymmetries in ${ }^{18} \mathrm{~F}$ as a function of integration width, deduced from the master spectrum. Integration limits are defined in the caption of Fig. 7.

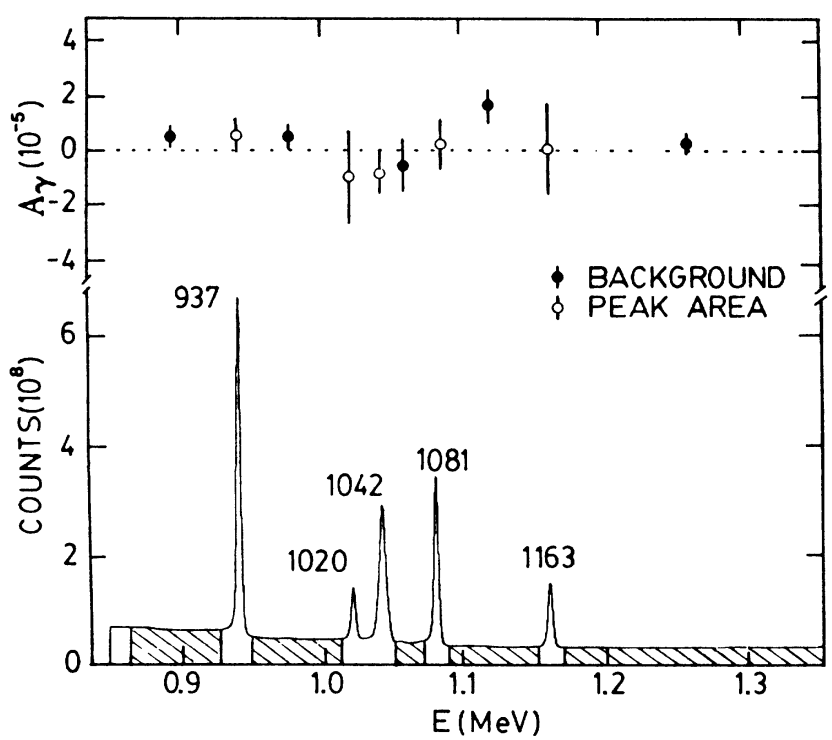

FIG. 9. A portion of the master spectrum form one detector, showing the calculated asymmetries of the five $\gamma$-ray peaks and background regions (shaded) from the $\pm 2.2 \sigma$ analysis. Error bars represent one standard deviation of the asymmetries. about the centroid of each $\gamma$-ray peak. The uncertainties $\delta A_{\gamma}$ are plotted as a function of integration width in Fig. 7 and are in agreement with the qualitative predictions discussed above. The corresponding asymmetries $A_{\gamma}$ are shown in Fig. 8 and are essentially independent of integration width provided that the narrowest windows are not chosen. The average optimum integration width for the five $\gamma$-ray corresponds to \pm 2.2 standard deviations $( \pm 2.2 \sigma)$ about the centroids of the $\gamma$-ray peaks. Polarimeter field dependent centroid shifts of the $\gamma$-ray peaks were less than $3 \times 10^{-4}$ channel; the difference between the peak asymmetries calculated from the master spectrum with and without a first order centroid shift correction to the peak areas was less than $10 \%$ of the statistical uncertainties $\delta A_{\gamma}$ at the optimum integration width.

As the results of the master spectrum analysis were in agreement with a preliminary analysis using a $\pm 3.5 \sigma$ integration width with no charge asymmetry rejection, the effect of the data selection appeared insignificant. The final analysis was performed with an integration width of $\pm 2.2 \sigma$ and a rejection criterion of $\left|A_{q}\right|>0.01$ which reduced the data base by $0.5 \%$. For each spectrum, centroids of the five $\gamma$-ray peaks were determined. Fixed background regions were defined with respect to the mean of the state (0) and state (1) centroids for a given peak. The average background in the peak regions was deduced by linear interpolation from the adjacent background regions. Fractional channel integration windows corresponding to $\pm 2.2 \sigma$ for each peak were set about the mean state (0)-state (1) centroid, and the total number of events in each peak and background region was determined. For each run, peak and background asymmetries were calculated from the eightfold ratios $\xi$ and were stored for further statistical analysis. The asymmetries of the peak areas and the background regions were calculated from the total number of events in these regions.

Results are plotted with a portion of the master spectrum in Fig. 9 and are summarized in Table II. The total number of $1.081 \mathrm{MeV}$ events was $1.5 \times 10^{10}$, and the peak to background area ratio was 3.7 , averaged over the four detectors. The peak asymmetries are consistent with zero, but there may be a small, positive asymmetry in the background regions. The asymmetries observed in the normal

TABLE II. The $\gamma$-ray asymmetries for peak areas and background regions deduced from the $\pm 2.2 \sigma$ data analysis described in the text. In the normal $(N)$ state the TS logic level (0) gave polarimeter state $(0)$ and in the reverse $(R)$ state logic level (1) gave polarimeter state $(0)$.

\begin{tabular}{cccc}
\hline $\mathrm{E}(\mathrm{keV})$ & $A_{\gamma} \times 10^{5}(\mathrm{~N})$ & $A_{\gamma} \times 10^{5}(\mathrm{R})$ & $A_{\gamma} \times 10^{5}$ (tot) \\
\hline $870-930$ & & & $0.48 \pm 0.42$ \\
937 & $1.34 \pm 0.92$ & $-0.07 \pm 0.92$ & $0.63 \pm 0.65$ \\
$945-1013$ & & & $0.48 \pm 0.52$ \\
1020 & $-2.5 \pm 3.0$ & $0.3 \pm 3.0$ & $-1.1 \pm 2.1$ \\
1042 & $-0.6 \pm 1.2$ & $-1.1 \pm 1.2$ & $-0.89 \pm 0.88$ \\
$1052-1073$ & & & $-0.63 \pm 0.95$ \\
1081 & $-1.5 \pm 1.3$ & $2.1 \pm 1.3$ & $0.27 \pm 0.93$ \\
$1089-1155$ & & & $1.56 \pm 0.61$ \\
1164 & $0.0 \pm 2.3$ & $-0.2 \pm 2.3$ & $-0.1 \pm 1.6$ \\
$1173-1365$ & & & $0.18 \pm 0.38$ \\
\hline \hline
\end{tabular}


$(N)$ and reverse $(R)$ polarimeter states as defined with respect to the logic levels are consistent with no statedependent systematic effects. The circular polarization of the $1.081 \mathrm{MeV} \gamma$ ray, deduced from the $\pm 2.2 \sigma$ analysis and the measured polarization sensitivity, is $P_{\gamma}(1.081)$ $=1.7 \pm 5.8 \times 10^{-4}$, where the uncertainty represents one standard deviation in the measurements. The $3 \%$ difference from the value quoted in an earlier publication ${ }^{17}$ is due to a reevaluation of the polarimeter sensitivity data.

\section{B. Evaluation of possible systematic effects}

The experimental circumstances, apart from the direction of the magnetic fields in the polarimeter cores, are not exactly identical in the two polarimeter states. Hence, it is necessary to examine whether corrections must be made to the eightfold ratio $\xi$, to isolate the contribution of the circular polarization to the observed $\gamma$-ray asymmetry. In general, the earlier equation for $\xi$ should be replaced by

$$
\xi=\frac{U(0) D(0) L(1) R(1)}{U(1) D(1) L(0) R(0)}=\left(\frac{1-A_{\gamma}}{1+A_{\gamma}}\right)^{4} \Omega_{c} D_{c} C_{c},
$$

where the correction factors $\Omega_{c}, D_{c}$, and $C_{c}$ account for differences in solid angle due to systematic beam motion, deadtime differences, and collimation effects for the two polarimeter states.

The solid angle correction factor $\Omega_{c}$ depends on $\Delta x$ and $\Delta y$, which are the vertical and horizontal amplitudes of systematic beam motion, and the coordinates $\delta x$ and $\delta y$, which describe the offset of the beam spot from the center of the polarimeter assembly. To first order in $\Delta x$ and $\Delta y$, $\Omega_{c}$ is given by

$$
\left|\Omega_{c}\right|=1+\frac{8}{r^{2}}(\Delta x \delta x-\Delta y \delta y),
$$

where $r$ is the target to detector distance. $\Delta y$ and $\Delta x$ can be determined from the horizontal and vertical steering ratios $R_{L R}$ and $R_{U D}$, which are given by

$$
\begin{aligned}
& R_{U D} \frac{U(0) D(1)}{U(1) D(0)} \simeq\left(1+8 \frac{\Delta x}{r}\right), \\
& R_{L R}=\frac{L(0) R(1)}{L(1) R(0)} \simeq\left(1-8 \frac{\Delta y}{r}\right) .
\end{aligned}
$$

The weighted averages of the vertical and horizontal steering ratios for the five $\gamma$-ray peaks are $R_{U D}$ $=1.000023 \pm 0.000024$ and $R_{L R}=1.000005 \pm 0.000025$; both are consistent with unity, indicating no significant beam motion effects. The upper limits (at one standard deviation) of systematic vertical and horizontal beam displacement amplitudes, set by these ratios, are $\simeq 10^{-6} \mathrm{~m}$; the predicted beam motion from the residual stray polarimeter fields is two orders of magnitude smaller. The values of these steering ratios imply a beam steering correction $\left|1-\Omega_{c}\right| \leq 10^{-6}$, which is negligible compared to the statistical uncertainty of the measured asymmetries $\delta A_{\gamma} \simeq 10^{-5}$.

The dead time correction factor $D_{c}$ is unity if all detector-amplifier systems have equal deadtime. $D_{c}$ may be expressed as

$$
D_{c}=\frac{\epsilon(U, 0) \epsilon(D, 0) \epsilon(L, 1) \epsilon(R, 1)}{\epsilon(U, 1) \epsilon(D, 1) \epsilon(L, 0) \epsilon(R, 0)},
$$

where $\epsilon(i, j)$ is the relative efficiency for detecting an event in detector $i$ in polarimeter state $j$. To evaluate $D_{c}$, these efficiencies must be known as a function of counting rate; measurements of the rate dependent detector efficiencies are described in Sec. IV A.

The collimation factor $C_{c}$ is unity unless systematic beam motion, possibly coupled with a substantial beam position offset, is sufficiently large that $\gamma$ rays from the target which would normally pass through the entrance collimators are differently blocked in the two polarimeter states. This situation did not arise during ${ }^{18} \mathrm{~F}$ data runs but was encountered during enhanced beam motion tests which will be discussed in Sec. IV B.

The weighted average of the charge dependent ratio $R_{Q}$ for all five $\gamma$-ray lines is $1.000198 \pm 0.000035$, where $R_{Q}$ is given by

$$
\begin{aligned}
R_{Q} & =\frac{U(0) D(0) L(0) R(0)}{U(1) D(1) L(1) R(1)} \\
& =\left[\frac{Q(0) t^{\prime}(0)}{Q(1) t^{\prime}(1)}\right]^{4} \frac{\epsilon(U, 0) \epsilon(D, 0) \epsilon(L, 0) \epsilon(R, 0)}{\epsilon(U, 1) \epsilon(D, 1) \epsilon(L, 1) \epsilon(R, 1)} .
\end{aligned}
$$

The effective counting time $t^{\prime}$ in one polarimeter state is given by

$$
\frac{t^{\prime}}{t}=\left(1-\frac{N_{\mathrm{MCS}}}{N_{\mathrm{SCA}}}\right),
$$

where $N_{\text {MCs }}$ is the number of events lost at the MUX due to ADC deadtime and random MUX failures, and $N_{\mathrm{SCA}}$ is the total number of events selected for conversion. The ratio of effective counting times can deviate significantly from unity due to random MUX failures, which lead to the loss of all incoming data for a fraction of a $10 \mathrm{~s}$ counting interval. MUX failures do not influence the $\gamma$ ray asymmetries, as the eightfold ratio $\xi$ is independent of the state (0) and state (1) counting times, but the MUX failures do contribute significantly to the deviation of the $R_{Q}$ from unity. To confirm the insensitivity of the asymmetries to deviations in $R_{Q}$ a correlation analysis was performed. For a sample of 4000 runs (30\% of the data), the $R_{Q}$ to $\xi$ covariance was less than $6 \times 10^{-4}$ for all peaks, indicating no significant correlation. Therefore, the apparent systematic asymmetry in the charge ratio $R_{Q}$ is concluded to have a negligible affect on the $\gamma$-ray asymmetries.

An internal test of spurious asymmetries is provided by other $\gamma$ rays produced in the reaction. The $1.042 \mathrm{MeV} \gamma$ ray has an isotropic angular distribution and negligible circular polarization. The other $\gamma$ rays in the spectrum are expected to have zero circular polarization, but not isotropic angular distributions so that magnetic-fieldinduced nuclear rotations could give rise to asymmetries if combined with detector misalignment. The maximum value of this misalignment relative to the beam direction is estimated to be $1^{\circ}$ and the amplitude of the stray magnetic field at the target position has been measured to be 
$5 \times 10^{-5} \mathrm{~T}$. If it is assumed that a nuclear state has a gyromagnetic ratio $g=1$ and a $\gamma$-ray angular distribution proportional to $1+b_{2} \cos ^{2} \theta$ ( $\theta$ is the angle between the ${ }^{3} \mathrm{He}$ beam direction and the outgoing $\gamma$ ray) with $b_{2}=1.0$, the asymmetry observed due to this effect in one polarimeter would have an upper limit of $8 \times 10^{-8} \tau$, where $\tau$ is the mean life of the nuclear state in ns. As $\tau \ll<1$ ns for all the populated states ${ }^{16}$ in ${ }^{18} \mathrm{~F}$, with the exception of the $\left(5^{+}, 1.21 \mathrm{MeV}\right)$ state, the 1.020 and $1.164 \mathrm{MeV} \gamma$ rays have negligible asymmetry. The $\left(3^{+}, 0.937 \mathrm{MeV}\right)$ state is populated by the decay from the $1.121 \mathrm{MeV}$ state which has $\tau=218 \pm 8$ ns (Ref. 18) and $g=0.572 \pm 0.006$ (Ref. 19). Measurements ${ }^{9}$ of angular distribution coefficients yield $b_{2}=0.75$ and, combined with the above data, predict an asymmetry of $7 \times 10^{-6}$ if it is assumed that the 0.937 $\mathrm{MeV} \gamma$ ray is only produced following the decay of the $1.121 \mathrm{MeV}$ state. However, this asymmetry must be attenuated by the $\gamma$-ray intensity ratio $I(1.121 \rightarrow 0.937$ $\mathrm{MeV}) / I(0.937 \rightarrow 0.000 \mathrm{MeV})$, which has been measured ${ }^{9}$ to be $8 \%$. Thus, for the $0.937 \mathrm{MeV} \gamma$ ray, the rotation induced asymmetry is also expected to be small compared with the statistical uncertainty in the circular polarization measurement. The average asymmetry of all $\gamma$ rays, excluding the $1.081 \mathrm{MeV}$ peak, in the observed portion of the spectrum, is $(0.01 \pm 0.48) \times 10^{-5}$. This result is a useful confirmation of the symmetry of the experimental equipment.

An additional test of the data analysis is the requirement that the calculated uncertainties accurately describe
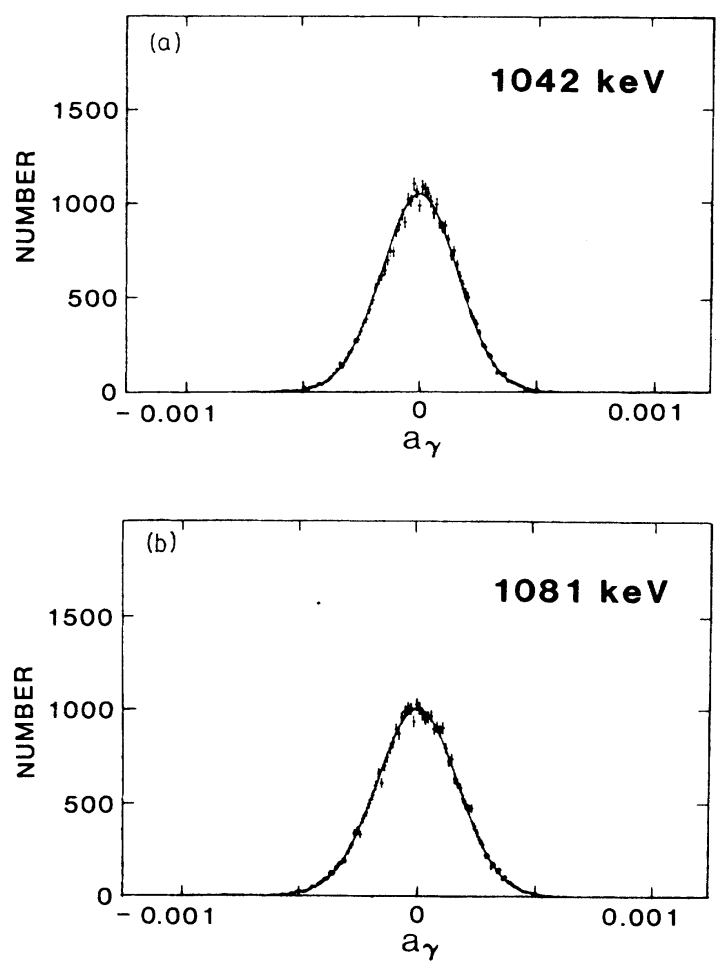

FIG. 10. Distribution of individual run asymmetries for the 1.042 and $1.081 \mathrm{MeV} \gamma$ rays from ${ }^{18} \mathrm{~F}$. The bin size is $\Delta a_{\gamma}=0.000125$. Solid lines are least-squares fits to a Gaussian function.
TABLE III. The ratios of the standard deviations $\sigma_{s}$ of the distribution of $a_{\gamma}$ to the statistical uncertainty $\delta a_{\gamma}$ in individual runs.

\begin{tabular}{cc}
\hline \hline$\gamma$ ray $(\mathrm{keV})$ & $\sigma_{s} / \delta a_{\gamma}$ \\
\hline 937 & 1.008 \\
1020 & 1.019 \\
1042 & 1.001 \\
1081 & 1.004 \\
1164 & 1.002 \\
\hline \hline
\end{tabular}

fluctuations in the individual asymmetry measurements $a_{\gamma}$ ( $\simeq 34000$ values $=11600$ runs $\times 3$ ADC's $)$. Histograms of the asymmetries for the 1.042 and $1.081 \mathrm{MeV} \gamma$ rays, calculated for individual runs, are shown in Fig. 10. The solid curves represent least-squares fits to the data with a Gaussian function. The standard deviations of these distributions are consistent with the statistical uncertainties for the individual runs, to within $0.5 \%$; details of this analysis are found in Table III.

\section{ENHANCED SYSTEMATICS TESTS}

Several auxiliary experiments were carried out to determine the sensitivity of the ${ }^{18} \mathrm{~F}$ circular polarization measurement to systematic variations of the ${ }^{3} \mathrm{He}^{+}$beam intensity and position on the target. These experiments were based on the premise that for sufficiently small variations of these parameters with the polarimeters turned off, the observed $\gamma$-ray asymmetries could be expressed as

$$
A_{\gamma}=\frac{\partial A_{\gamma}}{\partial \alpha_{i}} \delta \alpha_{i}
$$

and that a measurement of $A_{\gamma}$ with one parameter systematically varied would provide a value for the derivative $\partial A_{\gamma} / \partial \alpha_{i}$. From this information and the limits on systematic beam position and intensity modulations deduced from the circular polarization data, the magnitude of systematic correction factors can be deduced.

\section{A. Rate dependent effects}

The influence of count rate differences on the $\gamma$-ray asymmetries in ${ }^{18} \mathrm{~F}$ was investigated in two ways. The detector efficiencies were measured as a function of counting rate in order to calculate the dead time correction factor $D_{c}$, and an experiment was performed in which a small charge asymmetry $\boldsymbol{A}_{q}$ was induced in order to measure $\partial A_{\gamma} / \partial A_{q}$, from which $D_{c}$ could be inferred.

For the detector efficiency experiments, the $1.081 \mathrm{MeV}$ event rate in an additional $\mathrm{Ge}$ monitor detector $1 \mathrm{~m}$ downstream of the target was used as a measure of the input counting rate. The beam current was varied from 2 to $15 \mu \mathrm{A}$, and the $1.081 \mathrm{MeV}$ counting rates in the four $\mathrm{Ge}$ detectors were determined. After correcting for the dead time of the MUX unit, the $1.081 \mathrm{MeV}$ rates $\rho_{i}$, in detector $i$, were fitted to the function

$$
\rho_{i}=A_{i} Y \exp \left(-B_{i} Y\right),
$$


where $Y$ was the $1.081 \mathrm{MeV} \gamma$-ray rate observed in the monitor detector, the $A_{i}$ 's were normalization constants, and the constants $B_{U}, B_{D}, B_{L}$, and $B_{R}$ were fitted to be $3.26,3.08,3.23$, and $3.52 \mathrm{~ms}$, respectively. In the actual $\gamma$-ray circular polarization measurement the equivalent rate $Y$ was approximately 113 counts/s. The dead time correction factor $D_{c}$ is given by

$$
\begin{aligned}
D_{c} & =\frac{\epsilon(U, 0) \epsilon(D, 0) \epsilon(L, 1) \epsilon(R, 1)}{\epsilon(U, 1) \epsilon(D, 1) \epsilon(L, 0) \epsilon(R, 0)} \\
& =\exp \left[-\left(Y_{0}-Y_{1}\right)\left(B_{U}+B_{D}-B_{L}-B_{R}\right)\right],
\end{aligned}
$$

where $Y_{0}$ and $Y_{1}$ are the production rates in magnetization states 0 and 1 . The dead time correction is unity if $Y_{0}=Y_{1}$ or if $B_{U}+B_{D}=B_{L}+B_{R}$. From the fitted parameters, the ratio $\left(B_{U}+B_{D}\right) /\left(B_{L}+B_{R}\right)=0.939 \pm 0.005$, which indicates that the dead time correction cannot be ignored without further investigation.

To study the influence of count rate asymmetries on the circular polarization measurement a tungsten wire $(0.18$ $\mathrm{mm}$ diam, $12 \mathrm{~m}$ upstream from the target) was used to intercept a fraction of the beam synchronously with state (0)-state (1) data routing. Data were accumulated for 45 $h$ with the polarimeters turned off. The results were interpreted in two ways: Firstly, the observed asymmetry was interpreted as $A_{\gamma}=\left(\partial A_{\gamma} / \partial A_{q}\right) \delta A_{q}$; secondly, the ratios $\xi, R_{Q}, R_{U D}$, and $R_{L R}$ were predicted from the detection efficiency parameters and compared with observations.

The derivative $\partial A_{\gamma} / \partial A_{q}$ was measured to be $(-4.3 \pm 0.5) \times 10^{-3}$ for a charge asymmetry of $\delta A_{q}=-0.146$. For the ${ }^{18} \mathrm{~F}$ circular polarization data, the charge asymmetry was $A_{q}=-4.1 \times 10^{-5}$, which corresponds to an asymmetry offset $\delta A_{\gamma} \simeq 2 \times 10^{-7}$. As this charge-induced asymmetry is two orders of magnitude smaller than the statistical uncertainty $\delta A_{\gamma}$ for all the $\gamma$ ray lines from ${ }^{18} \mathrm{~F}$, the charge asymmetry correction is negligible.

To test the quantitative understanding of the dead time correction factor, the steering and polarization-sensitive ratios were calculated for the beam interruption experiment using the fitted time constants. The steering ratios were predicted to be $R_{U D}=1.0028 \pm 0.0007$ and $R_{L R}$ $=0.9955 \pm 0.0007$; the vertical steering ratio is in agreement with the experimental value of $1.0036 \pm 0.0004$, but the horizontal steering ratio prediction is smaller than the observed value, $R_{L R}=0.9973 \pm 0.0004$. This discrepancy could be attributed to a systematic centroid motion due to beam particles being absorbed asymmetrically by the wire with a displacement amplitude $\Delta y=0.04 \mathrm{~mm}$. This displacement is not unreasonably large, but unfortunately it cannot be estimated with sufficient accuracy from the dimensions of the wire, as the phase space of the beam particles is not known. Finally, the calculated polarizationdependent ratio was $\xi=0.9937 \pm 0.0010$, in agreement with the observed ratio $\xi=0.9949 \pm 0.0006$. Thus the rate-dependent systematic effects are well understood and the dead time correction to the ${ }^{18} \mathrm{~F} \gamma$-ray asymmetries is negligible.

\section{B. Systematic beam motion tests}

To study the influence of systematic beam motion on the $\gamma$-ray asymmetries, the polarimeters were turned off and the beam was deflected by a dipole magnet located 2 $m$ upstream from the target. The polarity of the current in this steering magnet was reversed every $10 \mathrm{~s}$ by the switching bridge and power supply which normally was used for the polarimeter magnets. The steering field was reversed in synch with the data routing by the TS controller. Initial tests showed that large beam deflections $(\sim 1 \mathrm{~mm})$ gave a combination of dead time effects due to steering induced changes in the beam current, $\gamma$-ray collimation effects because of beam motion perpendicular to the $L$ and $R$ detector axis, and solid angle effects resulting from the motion of the beam relative to the $U$ and $D$ detectors. To separate these effects the investigation was performed with as small deflections as possible $(\sim 0.1$ $\mathrm{mm}$ ), which still gave a significant measurement, and with steady beam currents (differing on average by less than $1 / 10^{4}$ in the two states).

In the beam motion experiment it was found that the average $\gamma$-ray peak vertical steering ration was $R_{U D}=0.9978 \pm 0.004$ and the average $\gamma$-ray peak asymmetry was $A_{\gamma}=(2.8 \pm 7.2) \times 10^{-5}$. This determines the slope $\partial A_{\gamma} / \partial R_{U D}=(1.3 \pm 3.3) \times 10^{-2}$, and when combined with the observed $R_{U D}=1.000023(24)$ for the circular polarization measurement results in the value $\delta A_{\gamma}$ $=(3 \pm 9) \times 10^{-7}$ for the uncertainty in $A_{\gamma}$ due to systematic beam motion. This result is an order of magnitude lower than statistical uncertainty in $A_{\gamma}$. It should be emphasized that the above estimate is deduced from an internal measurement of $R_{U D}$ based on counting rates in the actual experiment. From the measurements of the magnetic field along the beam axis due to the polarimeter magnets (Sec. II B) it is calculated that $\left|\left(1-R_{U D}\right)\right|$ $\sim 2 \times 10^{-6}$ and, consequently, that the steering-induced variation in $A_{\gamma}$ is two orders of magnitude less than the statistical uncertainty.

The magnetic field along the beam axis in the steering magnet was mapped with a Hall probe in the same way as the polarimeter external field was mapped (Sec. II B). A numerical integration program, which used the measured magnetic field values as input data, predicted a steering amplitude of $0.08 \mathrm{~mm}$ and thus $R_{U D}=0.9966 \pm 0.0009$, in agreement with the experimentally observed value quoted above.

\section{Background asymmetry investigation}

The analysis of the ${ }^{18} \mathrm{~F}$ circular polarization data suggests a small, positive asymmetry of the background regions which averages to $(0.5 \pm 0.2) \times 10^{-5}$. This asymmetry could be due to circularly polarized bremsstrahlung from relativistic positrons emitted in the decays of radioactive isotopes produced at the target. Evidence for production of both ${ }^{14} \mathrm{O}$ and ${ }^{15} \mathrm{O}$ from the reactions ${ }^{12} \mathrm{C}\left({ }^{3} \mathrm{He}, \mathrm{n}\right){ }^{14} \mathrm{O}$ and ${ }^{16} \mathrm{O}\left({ }^{3} \mathrm{He}, \alpha\right){ }^{15} \mathrm{O}$ was seen in the full $\gamma$ ray spectrum under experimental conditions. Both ${ }^{14} \mathrm{O}$ and ${ }^{15} \mathrm{O}$ decay by $\beta^{+}$emission with endpoint energies of 1.8 and $1.7 \mathrm{MeV}$ and half-lives of 71 and $122 \mathrm{~s}$, respectively. A separate experiment was carried out in which the water target was exposed to the ${ }^{3} \mathrm{He}^{+}$beam for 4 min and spectra were accumulated in the energy window $0.4-2.4 \mathrm{MeV}$ in three consecutive $2 \mathrm{~min}$ intervals with 
the beam off. Count rates were $1 \mathrm{~Hz}$ per detector in the energy window accepted for the ${ }^{18} \mathrm{~F}$ circular polarization measurement. Data were accumulated for $168 \mathrm{~min}$ in each interval. From these data the average background asymmetry in the ${ }^{18} \mathrm{~F}$ experiment is estimated to be $(0.4 \pm 0.3) \times 10^{-5}$, in qualitative agreement with the observed value.

\section{Summary}

The results of the enhanced systematics tests, together with the charge and steering ratios deduced from the circular polarization data, provide evidence that systematic beam position and intensity fluctuations do not significantly influence the measured $\gamma$-ray asymmetries in ${ }^{18} \mathrm{~F}$. From the charge asymmetry experiment, the rate dependent correction to $A_{\gamma}(1.081)$ was found to be two orders of magnitude smaller than the statistical uncertainty in the asymmetry itself. The controlled beam steering experiment confirmed that effects of systematic beam motion could be correctly predicted from accurate measurements of small magnetic fields along the beamline, supporting the prediction of negligible beam steering by residual fields from the polarimeters. From these experiments, and the statistical analysis of Sec. III B, it is concluded that the uncertainty in the $\gamma$-ray asymmetries is accurately described by the statistical error alone. From the measured asymmetry of the $1.081 \mathrm{MeV} \gamma$-ray line and the polarization sensitivity $\eta(1.081)=0.0161 \pm 0.0008$, the circular polarization of the $1.081 \mathrm{MeV} \gamma$ rays from ${ }^{18} \mathrm{~F}$ is deduced to be $P_{\gamma}(1.081)=(1.7 \pm 5.8) \times 10^{-4}$.

\section{v. CONCLUSIONS}

Based on the matrix element $\left\langle 0^{+}\left|V^{\mathrm{PNC}}\right| 0^{-}\right\rangle$calculated by Adelberger et al. ${ }^{5}$ and the theoretical prediction ${ }^{3}$ $f_{\pi}^{1} \geq 0$, the present experimental result $P_{\gamma}(1.081)$ $=(1.7 \pm 5.8) \times 10^{-4}$ constrains the weak pion-nucleon coupling strength $\left|f_{\pi}^{1}\right|=\left(0.4_{-0.4}^{+1.4}\right) \times 10^{-7}$. A summary of all ${ }^{18} \mathrm{~F}$ circular polarization measurements to date is given in Table IV. The weighted average $P_{\gamma}(1.081)$ $=(1.2 \pm 3.9) \times 10^{-4}$ yields a weak pion-nucleon coupling strength $\left|f_{\pi}^{1}\right|=\left(0.3_{-0.3}^{+1.0}\right) \times 10^{-7}$, approximately four standard deviations smaller than the DDH best value prediction $f_{\pi}^{1}=4.6 \times 10^{-7}$.

TABLE IV. Summary of ${ }^{18} \mathrm{~F}$ circular polarization measurements.

\begin{tabular}{lc}
\hline \multicolumn{1}{c}{ Author } & $P_{\gamma}(1081) \times 10^{4}$ \\
\hline Barnes et al. ${ }^{\mathrm{a}}$ & $-7 \pm 20$ \\
Bizzetti et al. ${ }^{\mathrm{b}}$ & $-4 \pm 30$ \\
Ahrens et al. ${ }^{\mathrm{c}}$ & $-10 \pm 18$ \\
Bini et al. ${ }^{\mathrm{d}}$ & $2.7 \pm 5.7$ \\
This work & $1.7 \pm 5.8$ \\
& \\
Weighted average $\left(\chi^{2}=0.68\right)$ & $1.2 \pm 3.9$ \\
\hline \hline
\end{tabular}

${ }^{\text {a Reference } 9 .}$

${ }^{\mathrm{b}}$ Reference 10.

${ }^{\mathrm{c}}$ Reference 11 .

${ }^{\mathrm{d}}$ Reference 12.
To put this result in perspective, it is worthwhile to consider the constraints imposed on $f_{\pi}^{1}$ by other experiments. In the two-nucleon system, only pp and np interactions have been studied to date; $p$ p experiments yield no information on $f_{\pi}^{1}$ as $\pi^{0}$ exchanges are excluded. In the np system, the angular asymmetry $A_{\gamma}$ in polarized thermal neutron capture $\overrightarrow{\mathrm{n}}+\mathrm{p} \rightarrow \mathrm{d}+\gamma$ is sensitive only to the isovector component of $V^{\text {PNC }}$. With matrix elements calculated by Adelberger and Haxton ${ }^{20}$ and the experimental result ${ }^{21} A_{\gamma}=(-4.7 \pm 4.7) \times 10^{-8}, f_{\pi}^{1}$ is deduced to be $(4.4 \pm 4.4) \times 10^{-7}$, which is in agreement with the result of the ${ }^{18} \mathrm{~F}$ experiments. A measurement of the longitudinal analyzing power in $\overrightarrow{\mathrm{p}}+\alpha$ scattering, sensitive to both isoscalar and isovector components of $V^{\mathrm{PNC}}$, has recently been reported re $^{2}$ to be $A_{z}=(-3.3 \pm 0.9) \times 10^{-7}$. This result is in agreement with a prediction based on DDH best values of the $\Delta T=0$ and $\Delta T=1$ coupling strengths.

Two other experiments have been performed on nuclei of the mass-20 region, ${ }^{19} \mathrm{~F}$ and ${ }^{21} \mathrm{Ne}$. Both cases are examples of two-level mixing between low-lying states whose wave functions are reasonably well understood. The PNC observables in ${ }^{19} \mathrm{~F}$ and ${ }^{21} \mathrm{Ne}$ depend on linear combinations of weak isoscalar and isovector coupling strengths. In ${ }^{19} \mathrm{~F}$, the asymmetry of the $110 \mathrm{keV} \gamma$ rays from the first excited state $\left(\frac{1}{2}^{-}, \frac{1}{2}\right)$, populated in the polarization transfer reaction ${ }^{22} \mathrm{Ne}(\overrightarrow{\mathrm{p}}, \alpha){ }^{19} \mathrm{~F}$, has been measured by two groups, ${ }^{23}$ and the weighted average is $A_{\gamma}(110)$ $=(-7.4 \pm 1.9) \times 10^{-5}$. In ${ }^{21} \mathrm{Ne}$, the circular polarization of the $2.789 \mathrm{MeV} \gamma$ ray from the $2.789 \mathrm{MeV}$ parity-mixed state $\left(\frac{1}{2}^{-}, \frac{1}{2}\right)$ was measured ${ }^{24}$ to be $P_{\gamma}(2.789)$ $=(0.8 \pm 1.4) \times 10^{-3}$. Both $P_{\gamma}(2.789)$ and $A_{\gamma}(110)$ can be expressed as $A f_{\pi}^{1}+B\left(h_{\rho}^{0}+0.58 h_{\omega}^{0}\right)$, where the coefficients $A$ and $B$ are matrix elements of PNC meson exchange operators. The coefficients $A$ and $B$ are deduced from matrix elements which have one third of the values of the $0+1 \hbar \omega$ Kuo results quoted in Table IV of Adelberger et al. ${ }^{5}$ This adjustment is justified in the case of the isovector matrix element in ${ }^{19} \mathrm{~F}$ as its value is constrained by the forbidden $\beta$ decay, ${ }^{19} \mathrm{Ne} \rightarrow{ }^{19} \mathrm{~F}(110 \mathrm{keV})$. However, in the case of the isoscalar matrix element in ${ }^{19} \mathrm{~F}$ and all matrix elements in ${ }^{21} \mathrm{Ne}$, this procedure is without firm foundation.

Constraints on the weak isovector and isoscalar coupling strengths deduced from these experiments and the PNC matrix elements are shown in Fig. 11. The ${ }^{18} \mathrm{~F},{ }^{19} \mathrm{~F}$, and $\vec{p}+\alpha$ results define a common overlapping region in which the weak isoscalar coupling is close to the DDH best value prediction. However, the long range isovector coupling strength $f_{\pi}^{1}$ is four standard deviations smaller than the DDH best value. The ${ }^{21} \mathrm{Ne}$ result does not coincide with the overlapping region defined by the three other experiments; this discrepancy may be due to the difficulty of calculating reliable PNC matrix elements in that nucleus.

Low energy $\overrightarrow{\mathrm{p}}+\mathrm{p}$ experiments, ${ }^{25,26}$ which are sensitive to several coupling constants, but not $f_{\pi}^{1}$, are consistent with the DDH best values. An experiment to measure the longitudinal analyzing power in $\vec{p}+{ }^{13} \mathrm{C}$ scattering is in progress at the University of Washington and is expect$\mathrm{ed}^{27}$ to determine pure isoscalar PNC effects. Another 


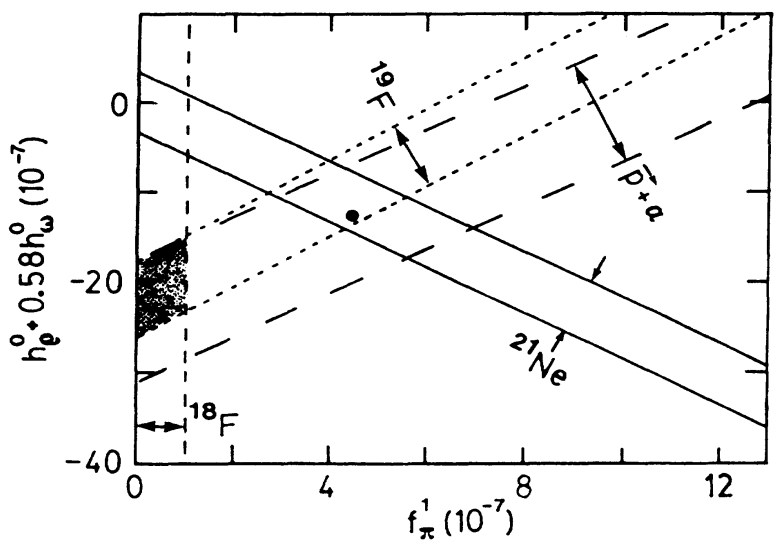

FIG. 11. Constraints on the weak isovector and isoscalar meson-nucleon coupling strengths deduced from PNC experiments in light nuclei and the matrix elements discussed in the text. All error bars represent one standard deviation. The solid circle is the DDH best value prediction. The shaded region indicates the range of parameters suggested by experiments, excluding ${ }^{21} \mathrm{Ne}$ (see text).

possible constraint to the isoscalar weak $\mathrm{N}-\mathrm{N}$ interaction might be obtained from a measurement of the longitudinal analyzing power in $\overrightarrow{\mathrm{d}}+\alpha$ elastic scattering. Predictions of the size of this effect have not yet been carried out.

The clear advantage of the ${ }^{18} \mathrm{~F}$ experiment is that it is sensitive to only the isovector component of $V^{\mathrm{PNC}}$, for which the nuclear matrix element can be accurately determined. The ${ }^{18} \mathrm{~F}$ results impose the most severe constraint on $f_{\pi}^{1}$ reported in the literature to date. It would be difficult to improve on the present measurement significantly. Based on the satisfactory performance of the Ti window foils, an improvement of $25 \%$ in the polarization sensitivity could be achieved by lengthening the polarimeters and increasing the beam current $20 \mu \mathrm{A}$. An order of magnitude improvement in the ${ }^{18} \mathrm{~F}$ measurement should be possible with the present apparatus, since systematic effects are estimated to be two orders of magnitude smaller than the present statistical uncertainty in the polarization measurement. However, the only apparent means of achieving such an improvement is to increase the data acquisition time to $\simeq 2 \times 10^{5} \mathrm{~h}$ with longer polarimeters. In view of the overwhelming uncertainties inherent in theoretical predictions of weak meson-nucleon coupling strengths, it is difficult to justify such an effort until theoretical calculations have been significantly improved.

Based on the results of recent experiments in ${ }^{18} \mathrm{~F}$, it would appear that, in spite of enhancement by weak neutral currents and strong interaction renormalization, the isovector component of the weak nucleon-nucleon interaction is suppressed relative to the isoscalar component. The implications of such a suppression have been discussed in detail by Donoghue and Holstein, ${ }^{28}$ who point out the strong restrictions imposed on quark models by small values of $f_{\pi}^{1}$. It is clear from this discussion that measurements of parity violation in nuclei have now reached the level of accuracy where they are imposing significant constraints on quark models used to calculate hadronic weak interactions.

\section{ACKNOWLEDGMENTS}

The authors wish to thank A. L. Bell, H. Janzen, and R. L. Stevenson of Queen's University for their many contributions to the experiment. The assistance of J. L. Gallant of Chalk River Nuclear Laboratories in the preparation of the target foils is greatly appreciated. The work was carried out with the help of grants from the Natural Sciences and Engineering Research Council of Canada. Further financial assistance was provided by Queen's University. Partial support to one of us (C.A.B.) was provided by the National Science Foundation under Grant Nos. PHY-82-15500 and PHY-85-08562 and to another of us (A.B.M.) by Grant No. PHY-86-045101.
*Present address: Department of Physics, University of Manitoba, Winnipeg, Manitoba, Canada R3T 2N2

${ }^{\dagger}$ Present address: Department of Modern Physics, China University of Science and Technology, Hefei, Anhwei, People's Republic of China.

$\ddagger$ Present address: TRIUMF, University of British Columbia, 4004 Wesbrook Mall, Vancouver, British Columbia, Canada V6T 2 A6.

${ }^{1}$ D. Tadic, Rep. Prog. Phys. 43, 67 (1980), and references therein.

${ }^{2}$ G. Barton, Nuovo Cimento 19, 512 (1961).

${ }^{3}$ B. Desplanques, J. F. Donoghue, and B. Holstein, Ann. Phys. (N.Y.) 124, 449 (1980).

${ }^{4}$ S. Weinberg, Phys. Rev. Lett. 19, 1264 (1967); A. Salam, in Elementary Particle Theory, edited by N. Svartholm (Almqvist and Wishells, Stockholm, 1968); S. Weinberg, Phys. Rev. D 5, 1412 (1972).

${ }^{5}$ E. G. Adelberger, M. M. Hindi, C. D. Hoyle, H. E. Swanson, and R. D. Von Lintig, Phys. Rev. C 27, 2833 (1983).
${ }^{6}$ A. E. Blaugrund, D. H. Youngblood, G. C. Morrison, and R. E. Segel, Phys. Rev. 158, 893 (1967); J. Keinonen, H.-B. Mak, P. Skensved, J. R. Leslie, and W. McLatchie, ibid. C 22, 351 (1980); J. Keinonen, H.-B. Mak, T. K. Alexander, G. C. Ball, W. G. Davies, J. S. Forster, and I. V. Mitchell, ibid. C 23, 2073 (1981); H. N. Catfor, E. F. Gorman, D. M. Pringle, and L. K. Fifield, Nucl. Phys. A407, 255 (1983).

${ }^{7}$ W. C. Haxton, Phys. Rev. Lett. 46, 698 (1981).

${ }^{8}$ A. M. Hernandez and W. W. Daehnick, Phys. Rev. C 25, 2957 (1982).

${ }^{9}$ C. A. Barnes, M. M. Lowry, J. M. Davidson, R. E. Marrs, F. B. Morinigo, B. Chang, E. G. Adelberger, and H. E. Swanson, Phys. Rev. Lett. 40, 840 (1978); M. M. Lowry, Ph.D. thesis, California Institute of Technology, 1978.

10P. G. Bizzeti, T. F. Fazzini, P. R. Maurenzig, A. Perego, G. Poggi, P. Sona, and N. Taccetti, Lett. Nuovo Cimento 29, 167 (1980).

${ }^{11}$ G. Ahrens, W. Harfst, J. R. Kass, E. V. Mason, H. Schober, G. Steffens, H. Waeffler, P. Bock, and K. Grotz, Nucl. Phys. 
A390, 486 (1982).

${ }^{12}$ M. Bini, T. F. Fazzini, G. Poggi, and N. Taccetti, Phys. Rev. Lett. 55, 795 (1985).

${ }^{13}$ S. B. Gunst and L. A. Page, Phys. Rev. 92, 970 (1953).

${ }^{14}$ E. A. Heighway, modified version of report by J. S. Colonais, Lawrence Berkeley Laboratory, Report UCRL-18439, 1968.

${ }^{15}$ T. F. Fazzini, P. R. Maurenzig, P. Sona, A. Stefanini, and N. Taccetti, Nucl. Instrum. Methods. 192, 287 (1982).

16P. Skensved, R. L. Stevenson, and H.-B. Mak (unpublished).

${ }^{17}$ H. C. Evans, G. T. Ewan, S.-P Kwan, J. R. Leslie, J. D. MacArthur, H.-B. Mak, W. McLatchie, S. A. Page, P. Skensved, S.-S. Wang, A. B. McDonald, C. A. Barnes, T. K. Alexander, and E. T. H. Clifford, Phys. Rev. Lett. 55, 791 (1985).

${ }^{18}$ F. Ajzenberg-Selove, Nucl. Phys. A392, 1 (1983).

${ }^{19}$ H. Schmidt, J. Morgenstern, H. J. Korner, J. Braunsfurth, and S. J. Skorka, Phys. Lett. 24B, 457 (1967).

${ }^{20}$ E. G. Adelberger and W. C. Haxton, Annu. Rev. Nucl. Sci. 35, 501 (1985).

${ }^{21}$ R. Wilson, M. Avenier, G. Bagieu, R. Hart, D. M. Koang, M. A. Idrissi, and B. Vignon, in The Investigation of Fundamental Interactions with Cold Neutrons, NBS Spec. Publ. No. 711, edited by G. L. Green (U.S. GPO, Washington, D.C., 1986), p. 85.

22J. Lang, Th. Maier, R. Muller, F. Nessi-Tedaldi, Th. Roser,
M. Simonius, J. Sromicki, and W. Haberli, Phys. Rev. Lett. 54, 170 (1985).

${ }^{23}$ K. Elsener, W. Grueller, J. Konig, P. A. Schmelzback, J. U1bricht, B. Vuaridal, D. Singy, C. Forstner, and W. Z. Zhang, Phys. Rev. Lett. 52, 1476 (1984).

${ }^{24}$ K. A. Snover, R. D. Von Lintig, E. G. Adelberger, H. E. Swanson, T. A. Trainor, A. B. McDonald, E. D. Earle, and C. A. Barnes, Phys. Rev. Lett. 41, 145 (1978); E. D. Earle, A. B. McDonald, E. G. Adelberger, K. A. Snover, H. E. Swanson, R. D. Von Lintig, H.-B. Mak, and C. A. Barnes, Nucl. Phys. A396, 221 1983).

${ }^{25}$ D. E. Nagle, J. D. Bowman, C. Hoffmann, J. L. McKibben, R. E. Mischke, J. M. Potter, H. Frauenfelder, and L. B. Sorenson, in High Energy Physics with Polarized Beams and Polarized Targets, Argonne 1978, AIP Proc. No. 51, edited by G. H. Thomas (AIP, New York, 1979), p. 24.

${ }^{26}$ R. Balzer, R. Henneck, Ch. Jacquemart, J. Long, F. NessiTedaldi, T. Roser, M. Simonius, W. Haeberli, S. Jaccord, W. Reichart, and Ch. Weddingen, Phys. Rev. C 30, 1409 (1984).

${ }^{27}$ E. G. Adelberger, P. Hoodbhoy, and B. A. Brown, Phys. Rev. C 30, 456 (1984).

28J. F. Donoghue and B. Holstein, Phys. Rev. Lett. 46, 1603 (1981); Phys. Rev. D 31, 70 (1985). 